\title{
Collective diffusion within the superionic regime of $\mathrm{Bi}_{2} \mathrm{O}_{3}$
}

\author{
Chris E. Mohn ${ }^{a *}$ \\ 1 Centre for Earth Evolution and Dynamics, \\ University of Oslo, N-0315 Oslo, Norway \\ Marcin Krynski ${ }^{b}$ \\ 2 Department of Chemistry, University of Cambridge, Cambridge, UK
}

(Dated: January 24, 2020)

The delta phase of $\mathrm{Bi}_{2} \mathrm{O}_{3}$ has the highest known value of oxide ion conductivity within the solid state and therefore remains a benchmark for the development of future generations of electrolyte materials to fuel cell technologies. Conventionally, the high value of conductivity in $\delta-\mathrm{Bi}_{2} \mathrm{O}_{3}$ has been explained by a large concentration of inherent vacancies, together with a strongly polarisable Bi-O bond. We show from ab initio molecular dynamics simulations that short "chains" of collective migrating oxygens contribute strongly to the high value of conductivity with the single particle Nernst-Einstein (N.E.) conductivity to collective (d.c.) conductivity $\sigma^{\text {N.E. }} / \sigma^{\text {d.c. }} \sim$ 0.5 at $1033 \mathrm{~K}$. The nature of collective events is investigated from a hopping model, the distinct part of the Van Hove function and from the extent of dynamical heterogeneities in the superionc regime. Results from this analysis indicates that the main contribution to collective ionic diffusion in $\delta-\mathrm{Bi}_{2} \mathrm{O}_{3}$ involves short collinear chains of 2 or 3 oxygens. These chains are either initiated by an oxygen that jumps into an already occupied oxygen cavity (where they co-exist for a very short time before the residential oxygen is kicked out of its cavity), or from a jump into a vacant cavity which trigger a next nearest neighbouring oxygen to migrate. Since $\delta-\mathrm{Bi}_{2} \mathrm{O}_{3}$ is easily stabilised in a range of environments, the nature of these collective chains can give important insight into the design of $\delta$ - $\mathrm{Bi}_{2} \mathrm{O}_{3}$-based fuel cells for the future.

Atomistic insight into the nature of ionic diffusion in the superionic regime has unveiled strong evidence for collective "multi ion" migration [1-13]. Although first suspected in 
the 1960s when Yokota showed that the popular Einstein relation failed to explain the discrepancy between the mobility and conductivity in silver-halides [14], it has not been until quite recently that atomistic simulations have shown that collective chains of migrating ions may possess lower energy barriers than those calculated from single-particle jumpdiffusion models $[2,3,8,15,16]$. Recent demonstrations include several Density Functional Theory (DFT) studies of highly mobile "chains" of $\mathrm{Li}^{+}$ions in sulphides and phosporites [6, 8], "string-like" movements of fluorines in fluorites [12] as well as 2D collective transport of oxygens in reduced perovskites [2]. Collective dynamics in these compounds are often advantageous since the nature of energetically preferred local motifs and their connectivity patterns are kept intact as the chain of ions migrate, minimizing bond-breaking and bondformation $[2,11]$. In the fast-ion conducting phase of strongly non-ideal $\mathrm{BaInO}_{2.50}$, for example, the vacancies are not distributed at random, but order on local and intermediate length-scale in corner shearing networks of $\mathrm{InO}_{4}, \mathrm{InO}_{5}$ and $\mathrm{InO}_{6}$ polyhedra. These motifs can only be kept intact if the ions migrate in a highly collective fashion. Single particle jumps would, in contrast, involve strongly repulsive O-O interactions and strained configurations, and the calculated activation energy for uncorrelated oxygen hops is therefore high [2]. Collective mechanism appear therefore to be advantageous over isolated jumps in non-ideal compounds where the ions are not randomly distributed over positions in a sublattice and therefore not "free" to jump to a random neighbouring cavity [15]. Bearing in mind recent effort in the search for new materials to operate Solid Oxide Fuel Cells (SOFCs) at lower temperature, capturing the nature of collective dynamics in fast-ion conductors can give useful input in the design and development of new electrolyte materials.

Superionic bismuth oxide, $\delta-\mathrm{Bi}_{2} \mathrm{O}_{3}$, has the highest known value of oxide ion conductivity within the solid state [17-19], and remains a benchmark for understanding ionic transport processes in the superionic regime. It adopts the cubic fluorite crystal structure (space group $F m \overline{3} m$ ), where the oxygens form a simple cubic anion sublattice and the bismuths occupies alternate cube centers (the $4 a$ site at $(0,0,0)$ ). The stoichiometry of $\delta$ - $\mathrm{Bi}_{2} \mathrm{O}_{3}$ implies that two of the eight anion cavities centered at the $8 c$ site at $1 / 4,1 / 4,1 / 4$ etc. surrounding each $\mathrm{Bi}^{3+}$ are vacant. This provides, together with a strongly polarisable and transient $\mathrm{Bi}-\mathrm{O}$ bond, a plausible explanation for the high value of ionic conductivity. Previous ab initio Molecular Dynamics (MD) calculations [20, 21] of the ionic conductivity used the Einstein relation, $\sigma^{N . E .}=\frac{\rho\left(e Z^{-}\right)^{2}}{k_{\mathrm{B}} T} D^{N . E}$, to calculate the conductivity from the diffusion coefficient, 
$D^{N . E}$. Here, $\rho^{-}$and $Z^{-}$are the density and the formal charge of the diffusing oxygens. The calculated Nernst-Einstein (N.E.) conductivity was found to be in good agreement with that obtained experimentally [17-19], which could indicate that collective diffusion does not contribute strongly to the overall ionic conductivity. On the other hand, previous studies have shown that the $\delta-\mathrm{Bi}_{2} \mathrm{O}_{3}$ is surprisingly non-ideal since only a fraction of the oxygenvacancy configurations are thermally available even at high temperature [20]. The oxygens, therefore, can not jump entirely at random to one of it's nearest neighbouring cavities since this could involve strongly repulsive vacancy vacancy interactions. This implies, in turn, that collective conductivity may be non-negligible for a critical comparison with conductivity measurements. Contribution from collinear chains of migrating oxygens may enhance the conductivity, and the good agreement between experiment and ab initio calculations could be fortuitous.

The extent of collective dynamics is often interpreted from the Haven ratio, $H_{R}=$ $D^{\text {N.E. }} / D^{\text {d.c. }}$, where $\mathrm{D}^{\text {N.E. }}$ is calculated directly from tracer-diffusion experiment and $\mathrm{D}^{\text {d.c. }}$ is estimated from conductivity experiment using the relation $\sigma^{\text {d.c. }}=\frac{\rho\left(e Z^{-}\right)^{2}}{k_{\mathrm{B}} T} D^{\text {d.c. }}$. Although the Haven ratio can be used to determine the nature of collective diffusion in the superionic regime, the dynamics of many superionic conductors with a high defect concentration is complex, and the extent of collective dynamics is difficult to interpret from the Haven ratio alone [22]. The advantage with ab initio MD simulations is that the nature of single-particle (tracer) correlation and collective dynamics can be investigated directly from the atomic trajectory which allows one to calculate the Haven ratio and compare directly with result from conductivity measurement.

In this work we will investigate atomistically different collective transport mechanism in $\delta$ - $\mathrm{Bi}_{2} \mathrm{O}_{3}$ using ab initio MD at the level of the generalized-gradient approximation to DFT. To our knowledge, understanding at the atomic level collective conductivity in $\delta$ - $\mathrm{Bi}_{2} \mathrm{O}_{3}$ and other fluorite-structured fast-ion oxide conductors remains unexplored, probably because much longer MD runs are needed to collect sufficient statistics in order to calculate the d.c. conductivity compared to that needed to calculate the (single particle) tracer diffusion coefficient. 


\section{THEORY}

The ionic conductivity itself is calculable by integrating the charge-current correlation function

$$
\sigma=\frac{1}{k_{\mathrm{B}} T V} \int_{0}^{\infty} J(t) \mathrm{d} t
$$

where $V$ is the volume and $J(t)=e^{2} \sum_{i j} Z_{i} Z_{j}\left\langle\mathbf{v}_{i}(0) \cdot \mathbf{v}_{j}(t)\right\rangle$, with $e, Z_{i}$ and $v_{i}$ being the elementary charge, the charge number and the velocity of ion $i$ respectively. To avoid the slow convergence in the integral over the charge current correlation function associated with non-negligible contribution from the tails in $J(t)$, we assume that we can ignore correlation between the velocities and re-express the conductivity in the form of a mean-square displacement [1]

$$
\sigma^{d . c .}=\frac{e^{2}}{k_{\mathrm{B}} T V} \lim _{t \rightarrow \infty} \frac{1}{6 t}\left\langle\left|\sum_{i} Z_{i}^{+} \delta \mathbf{r}_{i}^{+}(t)+\sum_{i} Z_{i}^{-} \delta \mathbf{r}_{i}^{-}(t)\right|^{2}\right\rangle .
$$

Here the summations within the brackets are the charge weighted net displacements of all cations and anions respectively. If we also ignore the correlations in the positions of the different ions in Eq. 0.2, we can write down the analogues (single-particle) Nernst-Einstein equation

$$
\sigma^{N . E .}=\frac{e^{2}}{k_{\mathrm{B}} T} \lim _{t \rightarrow \infty} \frac{1}{6 t}\left(\left\langle\rho^{+}\left|Z^{+} \delta \mathbf{r}^{+}(t)\right|^{2}\right\rangle+\left\langle\rho^{-}\left|Z^{-} \delta \mathbf{r}^{-}(t)\right|^{2}\right\rangle\right),
$$

where $\rho^{+}$and $\rho^{-}$are the densities of the cations and anions respectively. If we assume that the oxygen charge is a constant $\left(Z^{-}=-2\right)$ and that the cations do not diffuse (at least on the timescale of the MD simulation) we can calculate the d.c. conductivity from the Mean Square Displacement (MSD) of the centre of mass, C.M., of the mobile oxygen ions as:

$$
\sigma^{\text {d.c. }}=\frac{\left(e Z^{-}\right)^{2}}{k_{\mathrm{B}} T V} \lim _{t \rightarrow \infty} \frac{1}{6 t} \mathrm{MSD}^{\mathrm{C} . \mathrm{M} .}
$$

where $\mathrm{MSD}^{\mathrm{C} . \mathrm{M} .}=\left\langle\left|\sum_{i} \delta \mathbf{r}_{i}^{-}(t)\right|^{2}\right\rangle$ and similarly the N.E. conductivity

$$
\sigma^{N . E .}=\frac{\left(e Z^{-}\right)^{2} \rho^{-}}{k_{\mathrm{B}} T} \lim _{t \rightarrow \infty} \frac{1}{6 t} \mathrm{MSD}^{\text {N.E. }}
$$

with $\mathrm{MSD}^{N . E .}=\left\langle\left|\delta^{+}(t)\right|^{2}\right\rangle$ (where $\delta^{+}(t)$ is the average displacement of a single oxygen ion).

\section{COMPUTATIONAL DETAILS}

All results reported here are calculated using ab initio Born-Oppenheimer MD simulation in the NVT ensemble $\left(\mathrm{N}=270\right.$ atoms, $\mathrm{V}=(16.95 \AA)^{3}$ and $\left.\mathrm{T}=1033 \mathrm{~K}\right)$ with a time step 
of 4 fs. The volume was the same as those obtained from neutron diffraction experiment at 1033(3) K [23] and MD runs [20]. We use VASP [24-26] with the Perdew-Burke-Ernzerhof functional [24], an energy cut-off of $400 \mathrm{eV}$ for the electronic wave function and $605 \mathrm{eV}$ for the electronic charge density. Consistent with previous MD calculationss [20] the $6 \mathrm{~s}^{2} 6 \mathrm{p}^{3}$ and $2 \mathrm{~s}^{2} 2 \mathrm{p}^{4}$ electron configurations for the bismuths and oxygens were used and the Brillouin zone is sampled the gamma point only. In all MD runs we start by generating random oxygen positions by distributing oxygen/vacancies over the tetrahedral positions in the fluorite structure and in all runs statistics were collected for at least 150 ps, after about 20 ps of equilibration. This ensured sufficient statistics for the sampling of collective dynamics and thus the ionic conductivity after the ballistic regime i.e. from $t=1$ to $t=3$ ps.

\section{RESULTS}

The single-particle and collective mean square displacements are shown in Fig. 1. From $D^{\text {N.E. }}=\lim _{t \rightarrow \infty} \frac{1}{6 t} \mathrm{MSD}^{\text {N.E. }}$ and $D^{\text {d.c. }}=\lim _{t \rightarrow \infty} \frac{1}{6 t} \mathrm{MSD}^{\text {C.M. }}$ we find that $D^{N . E .}=8.810^{-6} \mathrm{~cm}^{2}$

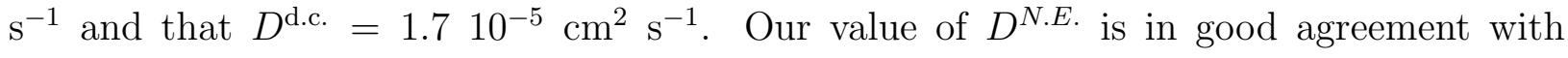
those found in previous MD runs [20,21] and also with that measured from tracer diffusion experiment $\left(D^{*}=110^{-5} \mathrm{~cm}^{2} \mathrm{~s}^{-1}\right)$ [27]. We find that the Nernst-Einstein conductivity, $\sigma^{N . E .}=2.7(\Omega \mathrm{cm})^{-1}$ at $1033 \mathrm{~K}$, is consistent with previous ab initio studies on superionic $\mathrm{Bi}_{2} \mathrm{O}_{3}$ at similar temperatures $[20,21]$, but $\sigma^{\text {d.c. }}$ is more than a factor 2 higher than that measured from d.c. conductivity experiment [17] (which is $\left.\sim 1.5(\Omega \mathrm{cm})^{-1}\right)$. Bearing in mind the large uncertainties associated with such conductivity experiment and that the approximation made to DFT as well as constraints imposed by periodic boundary conditions may influence ionic diffusion, the agreement is quite satisfactory.

We find that the Haven ratio, $H_{R}=0.51 \pm 0.04$, calculated using Eq. 0.2 and $H_{R}^{\text {dir }}$ calculated directly from Eq. 0.1 is $0.33 \pm 0.13$. The small discrepancy between these two values is probably due to the non-negligible contribution in the tails in $J(t)$ in Eq. 0.1 .

The low value of the Haven ratio is indicative for collective diffusion of oxygens, and to provide some mechanistic insight into the diffusion of the oxygens in $\delta-\mathrm{Bi}_{2} \mathrm{O}_{3}$, we first carry out a detailed atomistic analysis by calculating single particle and collective diffusion coefficient from a hopping model. We thus analyse characteristic times for hopping of isolated oxygens from an inspection of the ionic trajectories and then analyse the nature of collective 
events. Collective dynamics is analysed from dynamical heterogeneities in the simulation box by identifying atoms that move considerably distances - characteristic for interbasin jumps - in a small time interval. If these ions cluster together, the nature and size of these can help capturing the nature of collective excitation in the superionic regime.

\section{Single particle dynamics from a jump diffusion model}

We first analyse correlations between successive jumps of an oxygen by dividing the simulation cell into distinct space-filling primitive oxygen cubes [28-30] with the cubecenter located at the peak density position of the oxygens (i.e. at the tetrahedral $8 c$ site of $F m \overline{3} m)$ [31]. To distinguish vibrations with large amplitudes stretching into a neighbouring cavity and unsuccessful 'back jumps' (jump-relaxation), a jump is assumed to take place if the oxygen jumps from cavity "A" to "B" and stays within the new cavity for some time given by $\tau^{\text {thresh }}$. The value of $\tau^{\text {thresh }}$ should be chosen with some care because if $\tau^{\text {thresh }}$ is too large, i.e. on the size of the average residence time, $\sim \tau^{\text {residence }}$, two consecutive jumps may be identified as a single jump. If we set $\tau^{\text {thresh }}=1 \mathrm{ps}$, the calculated jump frequency is the same as that we find from an inspection of the ionic trajectories. Furthermore, the calculated diffusion using this threshold is in good agreement with that obtained from the $\mathrm{D}^{\text {N.E. }}$ which we turn to discuss below.

Results from this analysis shows that the average residence-time, $\tau^{\text {residence }}$, of an oxygen is slightly less than 10 ps with large vibrational amplitudes occurring dominantly in the $\langle 111\rangle$ direction. Although the peak position in the ionic density of the oxygens is at the centre of the cavity (i.e. at the $8 c$ site of $F m \overline{3} m$ ) [20], a typical oxygen position, when viewed locally, is shifted markedly in the direction of the octahedral hole in the crystallographic $\langle 111\rangle$ direction. We find that the oxygens migrate rapidly and decisively with $\tau^{\text {jump }} \sim 0.5-1.0$ ps. $90 \%$ of the jumps are between nearest neighbouring tetrahedral cavity aligned in the crystallographic $\langle 100\rangle$ direction whereas $\sim 10 \%$ of the jumps are between the next nearest neighbours aligned in the $\langle 110\rangle$ direction. Occasionally a jumping oxygens will carry out a short loop or wiggle near an interstitial octahedral position, but the octahedral site is not a residential site for the oxygen. In fact, it corresponds to an ionic density minimum [20], which also rules out an interstitial/intersitialcy diffusion mechanism for oxygen transport in superionic bismuth oxide. Of particular note is that we find that $20 \%$ of all jumps take 
place to an already occupied cavity which can initiate chains of collective migrating oxygens as discussed below.

Correlation between successive jumps, $f$, can be calculated using $f=1+2\left\langle\cos \theta_{l, l+1}\right\rangle$, where $\theta$ is the angle between jump $l$ and jump $l+1$. We then calculate $f$ by decompose two consecutive oxygen-jumps in the three distinct directions. Back-jumps (jump-relaxations) takes place when an atom jumps back to where it came from. Sideway jumps involves two consecutive jumps in different crystallographic directions whereas a forward jump describes an oxygen that jumps in the same direction as it did in the previous step, i.e. a jump in the $\langle 100\rangle$ direction is followed by a jump in $\langle 100\rangle$.

We find that $f$ is about 0.35 due to high high fraction of back-jumps which is also higher than that expected for a random diffusion process at $t>\tau^{\text {residence }}$. We can calculate the tracer-diffusion coefficient from a hopping model using: $\tilde{D}^{N . E .}=\frac{1}{6} f \Gamma a^{2}$, where $\Gamma$ and $a$ are the jump frequency and the hopping lengths. Inserting the values of $f, a$ and $\Gamma$ gives $\tilde{D}^{N . E .}=1.010^{-5} \mathrm{~cm}^{2} \mathrm{~s}^{-1}$ which is in good agreement with both $D^{N . E}$. calculated from the MSDs above (shown in Fig. 1), and with result from tracer-diffusion experiment reported in Ref. [27]. The good agreement between $\tilde{D}^{\text {N.E. }}$ calculated from a hopping model and $D^{\text {N.E. }}$ calculated from the MSD, gives some confidence in that a hopping model provide a useful tool to also analyse collective dynamics in the superionic regime.

\section{Collective dynamics from a pair hopping model}

We now investigate collective diffusion of oxygens along the MD trajectory from an inspection of correlation between pairs of jumping oxygens. We distinguish between events where the diffusion mechanism involves a common cavity (i.e. a "kick out" mechanism as shown in Fig. 2a), or if they do not involve a common cavity, as visualized in Fig. 2b. The "kick out" mechanism involves two oxygens, O1 and O2, that typically stay together in the same cavity, B, for a very short time (between 0.1 and $0.3 \mathrm{ps}$ ), before O2 is kicked out. Diffusion of an oxygen from cavity "A" to an empty cavity "B" can also "trigger" an oxygen in a neighbouring cavity, "C", to jump to cavity "D" (see Fig. 2b). We further distinguish between collinear, non-collinear and exchange mechanisms: If the cavities "A", "B" and "C" involved in the diffusion of the two oxygens are all aligned in the same crystallographic direction (as shown in Fig. 2a and Fig. 2b), the mechanism is termed collinear. If O1 jumps 
to cavity "B" while O2 jumps to cavity "A", the oxygens exchange positions which gives no net charge transport. A non-collinear mechanism involves cavities that are not aligned in the same crystallographic directions, as shown in Fig. 2c.

$20 \%$ of the oxygens jump into an already occupied cavity, and will therefore trigger collective chains of migrating oxygens. Of these, about $40 \%$ are involved in a collinear mechanism, $60 \%$ in a non-collinear path whereas less than $1 \%$ of these oxygens exchange positions. The fraction of collinear chains is thus substantially higher compared to that of a random diffusion process where the probability of collinear chains is $1 / 6$. This suggests that a collinear "kick out" mechanism could explain, at least in part, the low value of the calculated Haven ratio. We can quantify the contribution from a collinear "kick out" mechanism to the Haven ratio, by comparing the MSD of a "quasiparticle" of two oxygens involved in a collective chain with that when two oxygens hops at random on the oxygen sublattice. Since $40 \%$ of the $20 \%$ that jump into an already occupied cavity follow a collinear "kick out" mechanism, we can calculate the MSD from such a pair model from: $\mathrm{MSD}^{\text {kick out }}=\frac{1}{6} f \Gamma\left(P_{\text {vac }} \times a_{\text {pair random }}+P_{\text {kick out }} \times a_{\text {kick out }}\right)^{2}=$ $\frac{1}{6} f \Gamma\left(0.8 \times \sqrt{2} a_{n n}+0.2 \times(0.4 * 2+0.6 * \sqrt{2}) a_{n n}\right)^{2}$ where $P_{\text {vac. }}, P_{\text {kick out }}, a_{\text {random }}$ and $a_{\text {kick out }}$ are probabilities and hopping lengths for oxygens pairs that follows a (random) vacancy mechanism or kick out mechanisms respectively. The calculated Haven ratio from this pair model is therefore $H_{\mathrm{R}}^{\text {collinear kick out }} \approx \mathrm{MSD}^{\text {random }} / \mathrm{MSD}^{\text {kick out }}=0.9$, which is markedly higher than that calculated from the ratio of the $\mathrm{MSD}^{\text {N.E. }}$ to $\mathrm{MSD}^{\text {d.c. }}$. It is however worth bearing in mind that this model does not include contributions from migrating chains involving 3 or more oxygens. If we assume that chains of 3 migrating oxygens follow the same probability distribution as that of pairs, the Haven ratio calculated from a collinear kick out mechanism will decrease to about 0.8 . We do not expect a significant contribution to the Haven ratio from collective chains with more than 3 oxygens since the "kick out" chains are quickly terminated by a jump into a vacant cavity. This is also confirmed from an inspection of the trajectory which shows that chains involving more than 3 ions are rare. Collective collinear strings of 2 and 3 oxygens therefore contribute about $30 \%$ to all collective events.

The discrepancy between the Haven ratio calculated from $\mathrm{MSD}^{\text {N.E. }} / \mathrm{MSD}^{\text {d.c. }}$ and that estimated from a hopping model of pairs (and triplets) of migrating oxygens may, in part, be due to collective chains that have been averaged out in our analysis due difficulty in distinguishing vibrations and jumps. We have not yet included correlated events where 
pairs of oxygens do not stay within the same cavity "B" at the same time during diffusion, such as if O2 jumps to cavity "C" before O1 jumps to "B". Our jump diffusion model of oxygen pairs would identify such a collective oxygen pair as two uncorrelated jumps to vacant cavities. To estimate the contribution from such events to the Haven ratio we carried out an inspection of the oxygen trajectories which indicates that these events are rare. In addition, our analysis does not, so far, include events where the two cooperatively migrating oxygens are not nearest neighbours as shown in Fig. 2b. Such a mechanism was more important in superionic CuI than the "kick out" mechanism [31,32] and is not expected to contribute to the a first peak in $G_{d}^{O O}(r, t)$. We shall turn to investigate if such events contribute to the Haven ratio from the nature of the dynamical heterogneities.

\section{Collective diffusion from the distinct part of the Van Hove function}

The collinear "kick out" mechanism appear not to fully capture all collective events in $\delta-\mathrm{Bi}_{2} \mathrm{O}_{3}$ which can be further investigated from the distinct part Van Hove function $G_{d}^{O O}(r, t)=\frac{1}{N(N-1)} \sum_{i \neq j}^{N}\left\langle\delta\left(\left|\mathbf{r}_{i j}\left(t+t_{0}\right)-\mathbf{r}\left(t_{0}\right)\right|\right)\right\rangle$. If the kick out mechanism would account for all collective jumps, we might expect a peak to grow at short distances and times in the distinct part of the Van Hove function, $G_{d}^{O O}(r, t)$, as demonstrated for collective "kick out" chains of $\mathrm{Li}^{+}$ions in Li-based superionic conductors [6].

That is, since - in the kick out mechanism - cavity B is occupied by $\mathrm{O} 1$ even before $\mathrm{O} 2$ has left the cavity we expect a peak in $G_{d}^{O O}(\mathrm{r}, \mathrm{t})$ at times consistent with the characteristic jumptime of an oxygen $\left(\tau^{\text {jump }}=0.5 \mathrm{ps}\right)$ within intrabasin distances at $r<1 \AA$ and at $t \sim 0.5-1$ ps. However, as seen in Fig. 3, the peak position in $G_{d}^{O O}(r, t>2 \mathrm{ps})$ at $0.7-0.8 \AA$ is first visible at around $4 \mathrm{ps}$ which suggest that kick out events are not captured in $G_{d}^{O O}(r, t)$. The peak in $G_{d}^{O O}(r=0.7 \AA)$ therefore reveals little information about the collective diffusion of oxygens, but rather reflects the presence of large thermal anharmonic vibrations and a highly asymmetric local oxygen arrangement around the bismuth as discussed previously in Refs. [20, 23, 33]. 


\section{Collective diffusion from dynamical heterogeneity}

Challenges in capturing many collective events from a hopping model and the distinct part of the Van Hove function - due to difficulties in distinguishing vibrations with large amplitudes and jumps - has motivated us to investigate the nature of dynamical heterogeneity as a possible route to identify cooperative migrating oxygens. We therefore define the "rearrangement indicator" $R_{i}[34,35]$, of an oxygen, $i$, in order to measure the spacious region of its trajectory (within a small time window) as: $R_{i}=$ $\sqrt{\left.\left\langle\left(\mathbf{r}_{i}-<\mathbf{r}_{i}>_{t-\delta t / 2}\right)^{2}\right\rangle_{t+\delta t / 2}\left\langle\left(\mathbf{r}_{i}-<\mathbf{r}_{i}>_{t+\delta t / 2}\right)^{2}\right\rangle_{t-\delta t / 2}\right)}$ where $<>_{t+\delta t / 2}$ and $<>_{t-\delta t / 2}$ denotes time averages from $t$ to $t+\delta t / 2$ and from $t-\delta t / 2$ to $t$ with $\delta t=1$ ps. Note that this choice of $\delta t$ is the same as that used above for distinguishing vibrations with large amplitudes and ion hops $\left(\tau_{\text {thresh }}\right)$, but we stress that our results below are not strongly influenced by the choice of $\delta t$ as long as $0.1 \mathrm{ps}<\delta t<3$ ps. We identify oxygens that are likely to migrate or hop as those with $\sqrt{R_{i}}>0.95 a_{n n}$, (where $a_{n n}$ again is the distance between nearest neighbouring oxide ion positions). The oxygens that are moving a distance of a typical jump with time characteristic of a jump are marked as "red balls" in Fig 4, which shows 3 typical snapshots along an MD trajectory. It is evident that these oxygens form dynamical clusters and that these clusters can contain more than 2 oxygens! We can quantify the size of these clusters by counting all oxide ions with $\sqrt{R_{i}}>0.95 \mathrm{a}_{n n}$ that are within within a cut off distance, $d_{\max }=1.1 a_{n n}$ of an other "rearranging" oxygen with $\sqrt{R_{i}}>0.95 \mathrm{a}_{n n}$. This cut-off distance capture co-operative migrating groups of oxygens involving primarily nearest neighbouring atoms and result from this analysis shows that $14 \%$ and $3 \%$ of the clusters contain 2 and 3 (or more) oxygens respectively compared to $6 \%$ and less than $1 \%$ for a random distribution of red balls. If we increase $d_{\max }$ to $1.5 a_{n n}$ - and thereby include a larger fraction of oxygens involved in the mechanism shown in Fig $2 \mathrm{~b}$ - we find that $26 \%, 12 \%$ and $6 \%$ of the clusters contain 2,3 and more than 3 oxygens respectively. If we assume that the oxygens do not correlate we find that these numbers reduce to $19 \%$ and $4 \%$ for 2 and 3 atoms clusters. At an even higher cut-off distance $\left(d_{\max }=2.0 a_{n n}\right)$, there is a significant and higher fraction of three atoms clusters (18\%) compared to that of a random distribution $(12 \%)$. This indicates that oxygen clusters of next nearest and even third nearest neighbour are involved in collective diffusion of oxygens and if these jumps are in the same directions (e.g. as that shown in Fig $2 \mathrm{~b}$ ) they will contribute to the low value of the Haven ratio, $H_{R}$, 
The results form the cluster analysis are in good agreement with results from the hopping models discussed above. Both models indicate that about $20 \%$ of the oxygens jump to an already occupied cavity and are thus involved in a "kick out" mechanism. This suggests, in turn, that the rearranging oxygens are indeed oxygens that jump to new cavities and the shape and size of the clusters visualizes chains of migrating oxygens.

Results from hopping of pairs, the distinct part of the Van Hove function and the nature of dynamical heterogeneities show that diffusion processes in $\delta-\mathrm{Bi}_{2} \mathrm{O}_{3}$ is complex and include several distinct mechanism. Hopping to an already filled cavity can initiate collective colinear chains (see Fig. 2a) and hopping to vacant cavities can trigger hopping of an another oxygen that are not necessary a nearest neighbour (see Fig. 2b). The high inherent vacancy concentrations in $\delta-\mathrm{Bi}_{2} \mathrm{O}_{3}$ indicates that these strings are short, involving up to maximum four oxygens.

\section{Implication for the design of future solid oxide fuel cells}

The insight into collective diffusion from ab initio MD can provides us with essential input in the design and development of the next generations SOFCs. Although the $\delta$ phase of $\mathrm{Bi}_{2} \mathrm{O}_{3}$ itself is not a particular interesting candidate electrolyte materials for the use within SOFCs because of it's high temperature window of stability, it can easily be stabilized to lower temperature by aliovalent doping [36, 37], or as thin films grown on a suitable substrate $[38,39]$. This provide a range of opportunities to implement $\delta-\mathrm{Bi}_{2} \mathrm{O}_{3}$ within intermediate and low temperature fuel cell devices. Functionally graded bismuth oxide/ceria bilayers [40], for example, and ultrathin multilayers consisting of alternate $\operatorname{Er}_{2} \mathrm{O}_{3}$-doped $\mathrm{Bi}_{2} \mathrm{O}_{3}$ and $\mathrm{Gd}_{2} \mathrm{O}_{3}$-doped $\mathrm{CeO}_{2}$ sheets [41] both possess high chemical stability and high power densities at modest to low temperature. However, $\delta$ - $\mathrm{Bi}_{2} \mathrm{O}_{3}$ films grown directly on suitable substrates, such as $\mathrm{SrTiO}_{3}$, may relax to new phases [42] or can easily "crack up" forming dislocation misfits near the interface as shown from ab initio DFT calculations [43, 44]. Such a mismatch may increase the contribution from the $p$-type electronic conductivity to the total conductivity, but it is often extremely difficult to distinguish the electronic and ionic contributions to the conductivity in layered heterostructures [45-47].

Vertically grown architectures have challenged conventional lateral heterostructure for oxide fuel-cell technology, where nanorods and thin layers can be self assembled vertically 
on a suitable substrates [48]. This allows for a both high strain tunability - which is extremely difficult to achieve using $\delta$ - $\mathrm{Bi}_{2} \mathrm{O}_{3}$ thin-films grown layer-by-layer - and for a much better control of the contribution from the electronic currents to the conductivity. Indeed, Sm doped $\mathrm{CeO}_{2}$ electrolytes grown vertically as nanopillars on $\mathrm{SrTiO}_{3}$, show an order of magnitude higher oxide ion conductivity compared to plain Sm-doped films, with fast-ion diffusion occurring to large extent inside the nanopillars [48]. Collinar strings of cooperatively migrating oxygens can easily be accommodated within such architectures providing a possible new route to implement $\delta-\mathrm{Bi}_{2} \mathrm{O}_{3}$ as nanopillars/nanotubes to lower the current operation temperature of SOFCs.

\section{CONCLUSIONS}

We have carried out a detailed analysis of the nature of oxygen diffusion in the superionc phase of $\mathrm{Bi}_{2} \mathrm{O}_{3}$ using ab initio MD. The calculated Haven ratio, $H_{R}=D^{\text {N.E. }} / D^{\text {d.c. }}$, is markedly lower than 1 which indicates that collective chains of migrating oxygens contribute strongly to the d.c. conductivity. We explore the atomistic origin of these collective events from 1) a hopping model of oxygen "pairs" 2) the distinct part of the Van Hove function and 3) the nature of dynamical heterogeneities. We are unable to capture any collective chains from the functional form of $G_{d}^{O O}(r, t)$ but from a pair hopping model (where the number of oxygen hops in and out of their residential cavities are counted) we identify short collective chains of migrating oxygens that follow a "kick out" mechanism similar to that shown in Fig 2a. These chains are initiated by an oxygen that jumps into an already occupied cavity and then "kick out" the residential oxygen from that cavity. The calculated contribution to the Haven ratio from such collective two atom chains is only about $20 \%$, indicating that collective chains may contain at least three oxygens and/or that correlation length between different oxygen jumps may be longer than only involving nearest neighbours as, for example, cartooned in Fig 2b. To investigate this further we explore the nature of dynamical heteregeneities, where "clusters" of oxygens that move distances characteristic of typical a jump is identified. The nature of these "clusters" confirm that crystallographically collinear chains containing more than two atoms, also contribute to the ionic conductivity and that hops into vacant cavities can correlate with oxygen hops when these are aligned further apart than nearest neighbours. Results from hopping of pairs, the distinct part 
of the Van Hove function and the nature of dynamical heterogeneities show that the ion diffusion processes in $\delta$ - $\mathrm{Bi}_{2} \mathrm{O}_{3}$ is complex, blurred and involves different mechanisms which are not easily distinguished from one another. Nevertheless, it is evident that this complex dynamics plays an important role in promoting fast ion conductivity.

\section{ACKNOWLEDGEMENTS}

The Centre for Earth Evolution and Dynamics is funded by CoE-grant 223272 from the Research Council of Norway. The computing time was provided by the Norwegian metacenter for computational science (NOTUR) through a grant of computing time grant nn2916k. 
* Electronic address: chrism@geo.uio.no

[1] M. J. Castiglione and P. A. Madden, J. Phys.: Condens. Matter 13, 9963 (2001).

[2] C. E. Mohn, N. L. Allan, C. Freeman, P. Ravindran, and S. Stølen, Phys. Chem. Chem. Phys. 6, $3052(2004)$.

[3] F. Shimojo and M. Aniya, J. Phys. Soc. Japan 74, 1224 (2005).

[4] E. Kendrick, J. Kendrick, K. S. Knight, M. S. Islam, and P. R. Slater, Nature Mat. 6, 871 (2007).

[5] C. E. Mohn, N. L. Allan, C. L. Freeman, P. Ravindran, and S. Stølen, J. Solid State Chem. 178, 346 (2005).

[6] M. Xu, J. Ding, and E. Ma, Appl. Phys. Lett. 101, 031901 (2012).

[7] C. Tealdi, P. Mustarelli, and M. S. Islam, 20, 3874 (2010).

[8] X. He, Y. Zhu, and Y. Mo, Nat. Commun. 8, 15893 (2017).

[9] S. Hull, S. T. Norberg, S. G. Eriksson, and C. E. Mohn, J. Phys.: Condens. Matter 25, 454205 (2013).

[10] S. Stølen, E. Bakken, and C. E. Mohn, Phys. Chem. Chem. Phys. 8, 429 (2006).

[11] A. Vasileiadis and M. Wagemaker, cm 29, 1076 (2017).

[12] A. Annameareddy and J. Eapen, sr 7, 44149 (2017).

[13] A. Mascaro, Z. Wang, P. Hovington, Y. Miyahara, A. Poelella, V. Gariepy, Z. Feng, T. Enright, C. Aiken, K. Zaghib, et al., nl 717, 4489 (2017).

[14] I. Yokota, J. Phys. Soc. Japan 21, 420 (1966).

[15] N. L. Allan, S. Stølen, and C. E. Mohn, J. Mater. Chem. 18, 4124 (2008).

[16] X.-S. Kong, C. J. Hou, Q.-H. Hao, C. S. Liu, X. P. Wang, and Q. F. Fang, Solid State Ionics 180, 946 (2009).

[17] H. A. Harwig, Z. Anorg. Allg. Chem 444, 151 (1978).

[18] T. Takahashi, H. Iwahara, and Y. Nagai, J. Appl. Electrochem. 2, 97 (1972).

[19] T. Takahashi and H. Iwahara, J. Appl. Electrochem. 3, 65 (1973).

[20] C. E. Mohn, S. Stølen, S. T. Norberg, and S. Hull, Phys. Rev. B 80, 024205 (2009).

[21] A. Seko, Y. Koyama, A. Matsumoto, and I. Tanaka, J. Phys.: Condens. Matter 24, 475402 (2011). 
a)



II.

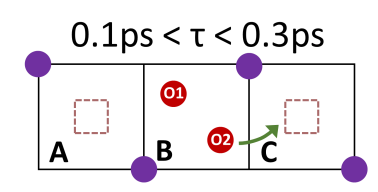

III.

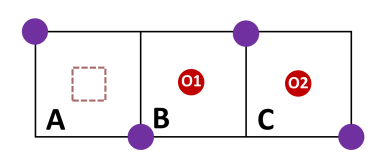

b)

I.



II.

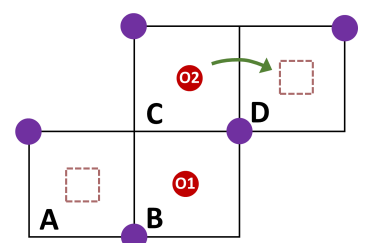

III.

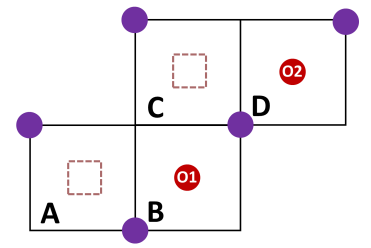

c)

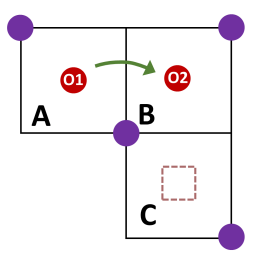

II.

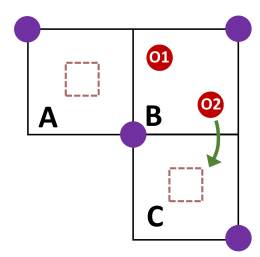

III.

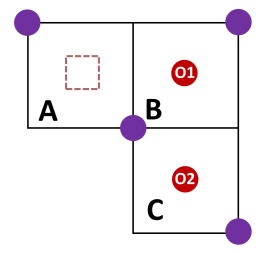

FIG. 2: The left picture (a) shows a cartoon of the collinear "kick out" mechanism where O1 and O2 shear a common cavity for a few vibrational periods before O2 is kicked out. Fig. 2b) is an example of a crystallographically collinear mechanism where O1 "trigger" an oxygen, O2, to jump in a neighbouring cavity during diffusion whereas c) is a non-collinear mechanism. 


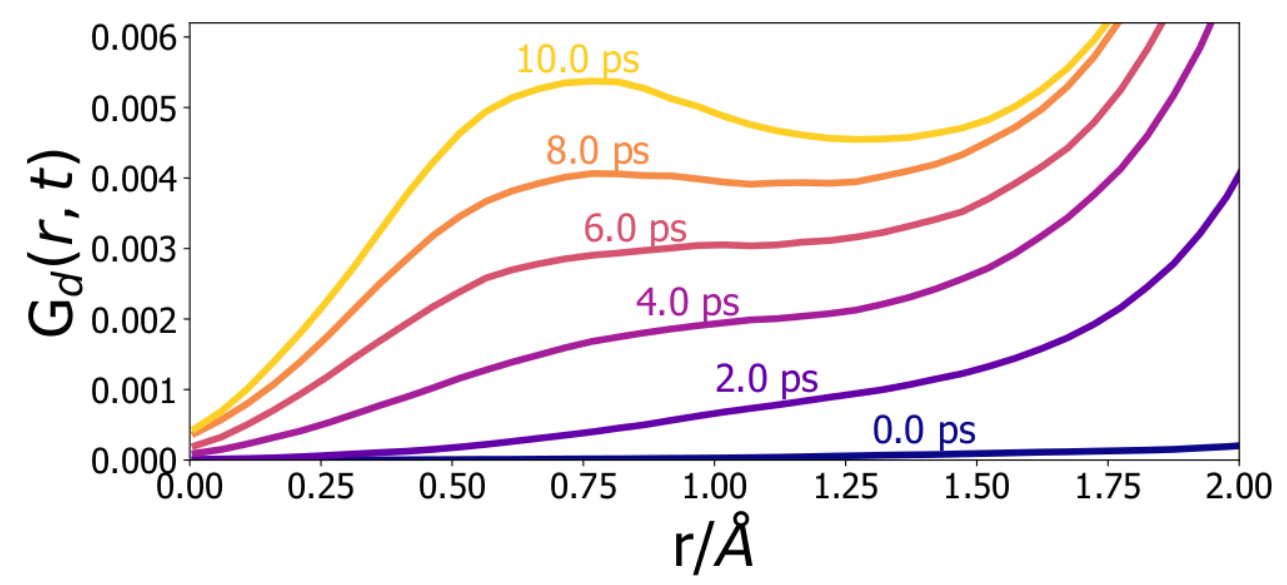

FIG. 3: Distinct part of the Van Hove correlation function of the oxygens $G_{d}^{O O}(r, t)=$ $\frac{1}{N(N-1)} \sum_{i \neq j}^{N}\left\langle\delta\left(\left|\mathbf{r}_{i j}\left(t+t_{0}\right)-\mathbf{r}\left(t_{0}\right)\right|\right)\right\rangle$ from ab initio MD runs at $1033 \mathrm{~K}$. The indexes $i$ and $j$ runs over all $N$ oxygens in the simulation box, and the brackets denotes averaging over different time origins, $t_{0}$.

[22] G. E. Murch, Solid State Ionics 7, 177 (1982).

[23] S. Hull, S. T. Norberg, M. G. Tucker, S. G. Eriksson, C. E. Mohn, and S. Stlen, Dalt. Trans. p. 8737 (2009).

[24] J. P. Perdew, K. Burke, and M. Ernzerhof, Phys. Rev. Lett. 77, 3865 (1996).

[25] G. Kresse and J. Hafner, Phys. Rev. B 47, 558 (1993).

[26] G. Kresse and J. Joubert, Phys. Rev. B 59, 1758 (1999).

[27] R. D. Bayliss, S. N. Cook, S. Kotsantonis, R. J. Chater, and J. A. Kilner, Adv. Energy Mat. 4, 1301575 (2014).

[28] G. Jacucci and A. Rahman, J. Chem. Phys. 69, 4117 (1978).

[29] S. Wengert, R. Nesper, W. Andreoni, and M. Parrinello, Phys. Rev. Lett. 77, 5083 (1996).

[30] C. E. Mohn, S. Stølen, and S. Hull, J. Phys. Soc. Japan 81, 106001 (2012).

[31] C. E. Mohn, S. Stølen, and S. Hull, J. Phys.: Condens. Matter 21, 335403 (2009).

[32] K. Tsumuraya, T. Ohtsuka, H. T. H. Oshihara, and M. Tsumuraya, J. Phys. Soc. Japan 81, $044603(2012)$.

[33] C. E. Mohn, S. Stølen, S. T. Norberg, and S. Hull, Phys. Rev. Lett. 102, 155502 (2009).

[34] R. Candelier, A. Widmer-Cooper, K. K. Kummerfeld, O. Dauchot, G. Biroli, P. Harrowell, 

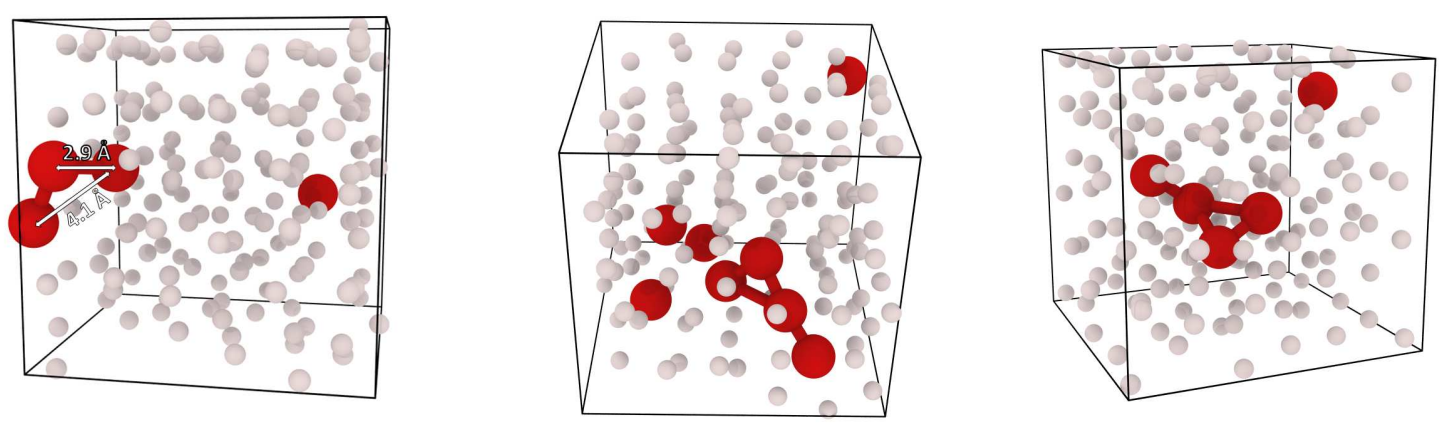

FIG. 4: Three random snapshots of oxide ion positions during an MD run at $1033 \mathrm{~K}$. The red spheres are oxygens with a rearrangement indicator, $\sqrt{R_{i}}>0.95 a_{n n}$, representing jumping oxygen ions, whereas the gray ones are those with $\sqrt{R_{i}}<0.95 a_{n n}$ (the non migrating oxygens). Sticks are drawn between red balls with $d_{\max }=1.5 a_{n n}$

and D. R. Reichman, Phys. Rev. Lett. 105, 135702 (2010).

[35] E. D. Cubuk, S. S. Schoenholz, E. Kaxiras, and A. J. Liu, 120, 6139 (2016).

[36] S. Hull, Rep. Prog. Phys. 67, 1233 (2004).

[37] P. Shuk, H. D. Wiemhöfer, U. Guth, W. Göpel, and M. Greenblatt, Solid State Ionics 89, 179 (1996).

[38] F. Tyholdt, H. Fjellvåg, A. E. Gunnæs, and A. Olsen, J. Appl. Phys. 102, 074108 (2007).

[39] K. Laurent, G. Y. Wang, S. Tusseau-Nenez, and Y. Leprince-Wang, Solid State Ionics 178, 1735 (2008).

[40] E. D. Wachsman, Science 334, 935 (2011).

[41] S. Sanna, V. Esposito, J. W. Andreasen, J. Helm, W. Zhang, T. Kasama, S. B. Simonsen, M. Christensen, S. Lindroth, and N. Pryds, Nature Mat. 14, 500 (2015).

[42] D. L. Proffit, G. R. Bai, D. D. Fong, T. T. Fister, S. O. Hruszkewycz, M. J. HIghland, P. M. Baldo, P. H. Fuoss, T. O. Mason, and J. A. Eastman, Appl. Phys. Lett. 96, 021905 (2010).

[43] C. E. Mohn, N. L. Allan, and S. Stølen, Nanosci. Nanotech Lett. 4, 178 (2012).

[44] C. E. Mohn, M. J. Stein, and N. L. Allan, J. Mater. Chem. 20, 10403 (2010).

[45] X. Guo, Science 324, 465a (2009).

[46] J. Garcia-Barriocanal, A. Rivera-Calzada, M. Varela, Z. Sefrioui, E. Iborra, C. Leon, S. J. Pennycook, and J. Santamaria, Science 324, 465b (2009). 
[47] A. Cavallaro, M. Burriel, J. Roqueta, A. Apostolidis, A. Bernardi, A. Tarancon, R. Srinivasan, S. N. Cook, H. L. Fraser, J. A. Kilner, et al., Solid State Ionics 181, 592 (2010).

[48] S. M-Yang, S. Lee, J. Jian, W. Zhang, P. Lu, Q. Jia, H. Wang, T. W. Noh, S. V. Kalinin, and J. L. MacManus-Driscoll, Nat. Commun. 6, 8588 (2015). 